\title{
Prevalencia de traumatismos dentales en deportistas del gimnasio Instituto Veracruzano del deporte
}

\section{Prevalence of dental trauma in athletes of the Veracruzano sports Institute gym}

HERNÁNDEZ-PASTRANA, Carlos Brandon†ं, CAPETILLO-HERNÁNDEZ, Guadalupe Rosalía*, TIBURCIO-MORTEO, Leticia y TORRES-CAPETILLO, Evelyn Guadalupe

ID $1^{\text {er }}$ Autor: Carlos Brandon, Hernández-Pastrana / ORC ID: 0000-0002-1015-3532

ID $1^{\text {er }}$ Coautor: Guadalupe Rosalía, Capetillo-Hernandez, ORC ID: 0000-0002-2033-4660, Researcher ID Thomson: S7875-2018, CVU CONACYT ID: 386320

ID $2^{\text {do }}$ Coautor: Leticia, Tiburcio-Morteo / ORC ID: 0000-0003-1806-4355

ID $3^{\text {er }}$ Coautor: Evelyn Guadalupe, Torres-Capetillo / ORC ID: 0000-0003-0576-0327, Researcher ID Thomson: T-16802018, CVU CONACYT ID: 308188

DOI: $10.35429 / J O H S .2019 .21 .6 .24 .27$

Recibido Octubre 10, 2019; Aceptado Diciembre 28, 2019

\begin{abstract}
Resumen
Introducción. Los deportistas están expuestos a traumatismos que en ocasiones puede estar involucrada la cavidad bucal desde una lesión, fractura o pérdida dental. Muchas veces conscientes o no que es un riesgo propio que representa practicar determinado deporte pero su vocación es mayor sin importar las consecuencias. Objetivos. Determinar la prevalencia de traumatismo dentales en deportistas entre 16-30 años del gimnasio Instituto Veracruzano del Deporte. Metodología. Estudio observacional, trasversal y prospectivo en una muestra de 43 deportistas aplicando una encuesta de 15 preguntas a deportistas del gimnasio Instituto Veracruzano del Deporte. Contribución. Fueron bajos los traumatismos dentales encontrados en esta investigación no obstante se debe de educar a los deportistas en la protección de accidentes mediante el equipo adecuado incluyendo la boca, implementando medidas preventivas obligatorias no sin antes concientizar sobre los cuidados de salud bucodental. Actualmente los traumatismos dentales son la segunda causa de atención en servicios de urgencia en odontología después de la caries.
\end{abstract}

Traumatismo dental, Deportistas, Prevalencia

\begin{abstract}
Athletes are exposed to trauma that can sometimes involve the oral cavity from an injury, fracture or dental loss. Many times aware or not that it is an own risk that represents practicing a certain sport but its vocation is greater regardless of the consequences. Objective. To determine the prevalence of dental trauma in athletes between 16-30 years of the gymnasium Institute Veracruzano of sport. Methodology Observational, cross-sectional and prospective study in a sample of 43 athletes applying a survey of 15 questions to athletes from the Institute Veracruzano of sport gymnasium. Contribution. The dental injuries found in this research were low, however, athletes should be educated in the protection of accidents through the appropriate equipment including the mouth, implementing mandatory preventive measures not before raising awareness about oral health care. Currently, dental injuries are the second cause of attention in emergency services in dentistry after caries.
\end{abstract}

Dental trauma, Athletes, Prevalence

Citación: HERNÁNDEZ-PASTRANA, Carlos Brandon, CAPETILLO-HERNÁNDEZ, Guadalupe Rosalía, TIBURCIOMORTEO, Leticia y TORRES-CAPETILLO, Evelyn Guadalupe. Prevalencia de traumatismos dentales en deportistas del gimnasio Instituto Veracruzano del deporte. Revista de Ciencias de la Salud. 2019. 6-21: 24-27.

\footnotetext{
*Correspondencia al Autor (Correo Electrónico: gcapetillo@uv.mx)

$\dagger$ Investigador contribuyendo como primer Autor
} 


\section{Introducción}

El trauma dentario es una lesión de extensión e intensidad variable, causada por fuerzas que actúan sobre el órgano dentario y los tejidos que le rodean y que puede ser observado y diagnosticado a simple vista o radiográficamente (Sánchez, 2016; González, 2011).

Los traumatismos dentales son eventos que suceden con frecuencia y por su gran impacto social y psicológico deben ser considerados como un tema de trascendental importancia

(Sánchez, 2016).

Las lesiones traumáticas bucodentales son afectaciones que se pueden encontrar en los jóvenes deportistas al estar expuestos al constante contacto al realizar el deporte, los odontólogos tienen la labor de inculcar en el joven deportista la importancia de la salud bucal ofreciendo medidas preventivas para una práctica deportiva segura y un tratamiento ideal después de recibir un trauma bucodental. (González, 2011).

Entre todas las enfermedades que afectan el complejo bucal se encuentran los traumas dentarios que son alteraciones causadas por factores extrínsecos e intrínsecos que dificultan el funcionamiento normal del mismo.(Matoses, 2015).

Los traumatismos dentales representan uno de los más serios problemas de salud pública entre niños y adolescentes. Esto se explica por la alta predominancia reportada en estudios poblacionales, los cuales presentan limitaciones debido a la ausencia de un estándar determinado para el examen y una clasificación definida de las lesiones. (Vantour, 2018).

El resultado de los traumatismos directos suele ser la lesión de los dientes anteriores, mientras que los indirectos favorecen la fractura de la corona y/o raíz de premolares y molares. Resulta importante señalar que, actualmente, más de $35 \%$ de los niños y adolescentes sufren traumas en sus dientes permanentes.
La educación para la salud es muy importante desde edades tempranas, en círculos infantiles, escuelas primarias y el hogar. Mediante entrevistas, charlas y otras técnicas, se debe llevar el mensaje de: la importancia de evitar el trauma y la necesidad de su atención y tratamiento inmediato. Los responsables de la educación para la salud bucal de los niños son los padres, estomatólogos, instituciones educacionales, al capacitarlos se influye de forma positiva en cuanto a la prevención del trauma dental insuficientemente tratado en la promoción de salud en estomatología.

La atención del paciente traumatizado generalmente ocurre en el servicio de urgencia, donde requiere de la dedicación profesional en la que debe primar el conocimiento y la actuación basada en las bases científicas y técnicas acordes al desarrollo actual. La prevención de este tipo de accidente puede considerarse una emergencia en salud pública y una emergencia social, debido a la elevada mortalidad y morbilidad dentaria que ocasionan, que traen como consecuencia un impacto negativo en la calidad de vida. (Sánchez, 2016).

Aunque hoy en día los traumatismos dentales son la segunda causa de atención odontopediátrica tras la caries, en un futuro muy cercano la incidencia de las lesiones traumáticas constituirá la principal demanda de atención.

\section{Metodología}

Estudio transversal, observacional y prospectivo. En los deportistas del gimnasio del Instituto Veracruzano del Deporte, en el periodo comprendido de febrero-julio 2019, con el objetivo de determinar la prevalencia de traumatismos dentales en los deportistas de ambos sexos con más 6 meses de actividad. De una muestra de 43 se trabajó con 33 deportistas de alto rendimiento a los que se les aplicó una encuesta de 15 preguntas y el apartado correspondiente a traumatismo dental del Formulario OMS de evaluación de la salud bucodentaL (1997) 


\section{Resultados}

En la encuesta realizada se preguntó a los deportistas si existían algunas molestias durante sus actividades en el gimnasio respondiendo el $85 \%$ de los encuestados que no sentían algún malestar, mientras que el $15 \%$ de los encuestados refirieron sentir molestias dentales durante la actividad y ellos mismos refirieron haber sufrido un traumatismo dental.

La medida preventiva que se puede usar al realizar actividades físicas en el gimnasio para protección de la cavidad oral, es el uso del protector bucal, el cual solo un $21 \%$ de los deportistas lo usan durante la práctica deportiva y un $79 \%$ no lo usan.

En nivel de conocimiento de las lesiones bucales que pueden ocurrir en los deportistas que realizan actividades deportivas en el gimnasio se muestra que el $36 \%$ tienen un conocimiento de estas lesiones mientras que el $64 \%$ tienen un nulo conocimiento sobre estas lesiones ocasionadas por las actividades deportivas realizadas.

La asistencia a la consulta dental al existir molestias durante los ejercicios realizados muestra que solo el $36 \%$ de los encuestados No asisten al dentista en caso de presentar molestias, mientras que el $64 \%$ sí.

La prevención de las actividades de los deportistas es de suma importancia para evitar las lesiones bucales, durante la encuesta se obtuvo que el $79 \%$ de los pacientes no usan guarda dental por lo cual se preguntó que si estarían dispuestos a utilizar guardas dentales para evitar lesiones y solo el $20 \%$ no usarían la guarda dental mientras que el $80 \%$ estaría dispuesto a usarla.

\section{Discusión}

Existen diversas investigaciones en donde se presentan resultados muy altos de prevalencia de traumatismos dentales en atletas de alto rendimiento sobre todo en halterofilia y atletismo son las áreas más afectadas.

En la presente investigación solo el 15\% de los que asisten al gimnasio IVD presentaron alguna vez una molestia dental y habían sufrido algún traumatismo dental.
De acuerdo a un estudio de la Universidad Nacional de Loja sobre lesiones bucales en deportistas por Gina Dayanna Ordoñez Ochoa las lesiones bucales que más se presentan en la cavidad bucal, son las contusiones (golpes) con un $43 \%$ de los casos, seguido por las laceraciones (cortes) bucales con un $18 \%$, y las fracturas dentales con un $14 \%$ tomando en consideración que el deporte que se abarco fue taekwondo, judo, boxeo, karate do y lucha libre, el cual presentan más contacto por ser un deporte en donde el objetivo es golpear a su contrincante

\section{Conclusiones}

Con base a esta investigación se puede llegar a la afirmación que la prevalencia de traumatismo dentales en el gimnasio Instituto Veracruzano del deporte es baja solo el $15 \%$ de los encuestado afirmo sentir molestias dentales y haber sufrido un traumatismo dental durante su rutina del gimnasio.

Cabe resaltar que al explicarles las ventajas del uso de una guarda dental durante la práctica del gimnasio el $80 \%$ estaría dispuesto a usar una guarda dental o protector dental en un futuro.

\section{Recomendaciones}

El gimnasio al ser como una actividad complementaria de otras disciplinas deportivas puede ser que no genere tantos traumatismos dentales. No obstante, sería buena la prevención mediante la implementación del uso de aparatos dentales idóneos durante la práctica deportiva.

\section{Referencias}

González YC. 2011Prevalencia de secuelas de traumatismos en dientes temporales en niños institucionalizados del municipio Playa. Revista Cubana de Estomatología..

Mallqui Herrada LLHAJF. 2012 Traumatismos dentales en dentición permanente. Revista Estomatológica Herediana. enero-marzo; XXII(1): p. 42.

Matoses VF. 2015Traumatismos dentales en Valencia. Tesis doctoral. Valencia: Universidad de Valencia, Facultad de medicina y odontología;. 
Sánchez TB. 2016Traumatismos dentarios en niños y adolescentes. Correo científico médico de Holghín..

Vantour DACL. 2018. Capacitación sobre traumatismos dentales en atletas adolescentes. MEDISAN. MARZO;: p. 234-235. 